\title{
Preparation of Neogambogic Acid Nanoliposomes and its Pharmacokinetics in Rats
}

\author{
Shi-juan Liu1, Jia-wei Liu33, Jun-zi Cong2, Lei Tong33, Ying Zhang1, Xue-mei Li³ and Jia-fu Hou³
}

\begin{abstract}
Objective: To prepare neogambogic acid nanoliposomes (GNA-NLC) and study its pharmacokinetics (PK) in rats.

Study Design: An experimental study.

Place and Duration of Study: Mudanjiang Medical University, Mudanjiang, China, from January 2016 to October 2017. Methodology: GNA-NLC was prepared by emulsion evaporation-low temperature solidification. The entrapment efficiency, average particle size, and zeta potential were investigated. Male Wistar rats were injected with $1 \mathrm{mg} / \mathrm{mL}$ gambogic acid and GNA-NLC into the caudal vein respectively, and the plasma concentration was determined by UPLCMS/MS. The pharmacokinetic parameters of the two agents were compared.

Results: GNA-NLC prepared in this study were mostly spherical spheroids with an average particle size of $146.35 \pm 1.72$ $\mathrm{nm}$, polydispersity coefficient of $0.26 \pm 0.02$, zeta potential of $-28.24 \pm 0.13 \mathrm{MV}$, entrapment efficiency of $84.63 \%$, and drug loading capacity of $4.23 \%$. DSC showed that neogambogic acid nanoparticles had formed and neogambogic acid was amorphous in the matrix. The pharmacokinetics results in rats showed that GNA-NLC plasma concentration was significantly higher than that of common preparation of gambogic acid, with a half-life period of $10.14 \pm 0.03$ hours, 4.57 times that of gambogic acid. AUC $0 \sim 24 \mathrm{~h}$ of gambogic acid in GNA-NLC lipidosome was $58.36 \pm 0.23 \mu \mathrm{g} / \mathrm{h} / \mathrm{mL}, 4.83$ times that of gambogic acid.

Conclusion: GNA-NLC can be prepared successfully by emulsion evaporation-low temperature solidification. The method is simple and easy to control. The GNA-NLC has a long cycle, and high blood concentration, sustained release compared with the raw material gambogic acid.
\end{abstract}

Key Words: Neogambogic acid nanostructured lipid carrier, Emulsion evaporation-low temperature solidification, Rat, Pharmacokinetic behaviour.

\section{INTRODUCTION}

Gamboge is a colloidal resin secreted by gamboge tree, garcinia maingayii. As a traditional Chinese medicine, gamboge is mainly used for attacking toxic, detumescence, removal of dental caries, closing sores, hemostasis, insecticide, etc. It is mainly used to treat ulcer, pyogenic infections, anabrosis, eczema, tumors, stubborn dermatitis, traumatic injury and hemorrhage. ${ }^{1}$ Neogambogic acid (GNA) monomer has been extracted from gamboges. Studies have confirmed that GNA is a major component for anti-tumor effects of gamboges, which can inhibit liver cancer HepG2 cells, lung cancer A549 cells, breast cancer cells and other tumor cells in varying degrees. ${ }^{2}$ It is not sensitive to normal cells like HUVEC cell lines, human umbilical vein endothelial

\footnotetext{
${ }^{1}$ Department of Pharmacy / Scientific Research ${ }^{2}$, Hongqi Hospital of Mudanjiang Medical University, Mudanjiang, Heilongjiang Province, 157011, China

3 Department of Pharmacology, Mudanjiang Medical University, Mudanjiang, Heilongjiang Province, 157011, China

Correspondence: Jia-fu Hou, Department of Pharmacology, Mudanjiang Medical University, Mudanjiang, Heilongjiang Province, 157011, China

E-mail:dsx345208066146@163.com

Received: January 27, 2018; Accepted: July 30, 2018
}

cells, and does not affect the spleen, kidney and other organ functions. However, GNA is a strong fat-soluble ingredient that is insoluble in water and freely soluble in organic solvents such as methanol, ethanol and acetone. GNA pharmacokinetic study found that, GNA can be rapidly eliminated from rats after intravenous administration with very short half-life period. ${ }^{3}$ These features lead to restricted GNA development and clinical application.

With the development of nanotechnology, the research of nanometer drug-loading system is also becoming more and more mature. Especially, in the field of anticancer, nanometer drug-loading system plays an indispensable and unique role. In particular, lipid nanoparticles (such as solid lipid nanoparticles, nanostructured lipid carriers, etc.) are widely studied. 4 Nanostructured lipid carrier (NLC) is a colloidal drug loading system with special nanostructures prepared by mixing solid lipids and liquid lipids that are spatially incompatible with each other at a certain temperature. Belonging to the field of aqueous dispersed system, it supports administration in a variety of ways to solve difficult drug delivery problems of many poorly water soluble medicines. ${ }^{5}$

At the same time, as the second generation of lipid nanostructured carrier system, NLC inherits various advantages of traditional nano-carriers (nanoemulsion, 
liposome, etc.) and solid lipid nanoparticles, 6 and has the additional advantages of high loading capacity, good stability, and sustained release on target. ${ }^{7}$

The purpose of this study was to prepare GNA-NLC and study its pharmacokinetics in rats for references of GNANLC in clinical practice.

\section{METHODOLOGY}

This study was carried out in Mudanjiang Medical University, China, from January 2016 to October 2017. The research was conducted after approval from the Committee on Animal Research and Ethics. The experiment prepared GNA-NLC by emulsion evaporationlow temperature solidification. The prescription dose of GMS, SA, MCT (the total mass of the three lipids is 200 $\mathrm{mg}$, mass fraction of MCT accounts for $20 \%$ of total lipid), lecithin (5.0 mg/mL mass concentration) and GNA was dissolved in $5 \mathrm{~mL}$ anhydrous ethanol, heated in water bath to $68 \sim 70^{\circ} \mathrm{C}$ to form an oil phase. Polysorbate-80, F68 at a ratio of 1: 1 was dissolved in 20 $\mathrm{mL}$ of water, heated in water bath to the same temperature as the oil phase. The oil phase was slowly and uniformly injected into a stirred (1000 $\mathrm{r} /$ minute) aqueous phase with a $1 \mathrm{~mL}$ syringe, and stirring was continued for 3 hours. After the organic solvent was completely volatilised, the emulsion was quickly poured into $40 \mathrm{~mL}$ of ice water (ice bath) while stirring, and stirring was continued for 2 hours to obtain GNA-NLC.

In this experiment, GNA-NLC entrapment efficiency was determined with reference to micro-column centrifugation in literature. ${ }^{8}$ GNA-NLC $100 \mu \mathrm{L}$ were loaded it on the top of the micro-column. After elution for six times with 0.3 $\mathrm{mL}$ distilled water added each time, continuously elution was done for 17 times. Filtrate containing GNA-NLC was collected in 1 6 tubes. Methanol was added to dissolution set to $25 \mathrm{~mL}$. The mass (M1) of encapsulated GNA in the nanoparticles was analysed and calculated by HPLC. Another GNA-NLC $100 \mu \mathrm{L}$ without column chromatography was added with methanol for dissolution and set to $25 \mathrm{~mL}$. By sample analysis, the total mass of GNA in the dispersed system (M0), entrapment efficiency and drug loading capacity were calculated.

Entrapment efficiency $=M_{1} / M_{0}$

Drug loading capacity $=W_{\text {administration }} \times$ entrapment efficiency $/$ $\left(W_{\text {administration }} \times\right.$ entrapment efficiency $\left.+W_{\text {fat }}\right)$

In formula (2), $W_{\text {administration }}$ is the total drug mass in the system; and $\mathrm{W}_{\text {fat }}$ is matrix mass. In this study, three batches of GNA-NLC were prepared by the same process, and their entrapment efficiency and drug loading capacity were measured.

Appropriate amount of GNA-NLC suspension was diluted by 30 times. Particle size and zeta potential were measured at room temperature using a zetasizer nanolaser particle analyser.
Five to six grams samples were placed in an aluminum pot subjected to scanning at a rate of $10^{\circ} \mathrm{C} / \mathrm{min}$ within a certain temperature range. With blank aluminum pot as a control, the pyrolysis curve of each sample was filled for DSC analysis using Origin 7.5 software.

The GNA-NLC was dissolved in ethanol to prepare a solution with a mass concentration of $100 \mathrm{mg} / \mathrm{mL}$, and then diluted to $1 \mathrm{mg} / \mathrm{kg}$ with PEG 400 . Twelve male Wistar rats were randomly divided into two groups. One group was treated by tail vein injection of $1 \mathrm{mg} / \mathrm{kg}$ gambogic acid solution and the other group was by injection of $1 \mathrm{mg} / \mathrm{kg}$ GNA-NLC solution. Then $0.2 \mathrm{~mL}$ blood was collected from rat orbit at $5,10,20,30$ minutes and at 1, 2, 4, 6, 8, 10, 12 and 24 hours after administration, added with heparin sodium anticoagulation and centrifuged at $2827 \times \mathrm{g}$ for 10 minutes. Plasma was separated and reserved. Fifty $\mu \mathrm{L}$ of plasma and $10 \mu \mathrm{L}$ of $300 \mathrm{ng} / \mathrm{mL}$ tripterine standard solution were mixed using vortex mixer for two minutes. One $\mathrm{mL}$ of ethyl acetate was added, followed by mixing using a vortex mixer for one minute, and centrifuging at $6,000 \mathrm{r}$ / minute for 10 minutes. The upper organic phase was taken, dried under nitrogen at $37^{\circ} \mathrm{C}$, redissolved in 100 $\mu \mathrm{L}$ of $50 \%$ methanol and centrifuged at $6,000 \mathrm{r} /$ minute for 10 minutes. Eighty $\mu \mathrm{L}$ samples were taken for detection. Plasma gambogic acid was determined by UPLC-MS/ MS method. The feasibility of UPLC-MS/MS method was validated with good specificity and no endogenous interference. The lowest limit of quantification was $5 \mathrm{ng} / \mathrm{mL}$. The standard curve was plotted with integral value of peak area of gambogic acid/integral value of peak area of tripterine as $Y$-axis $(Y)$, and with mass concentration of gambogic acid as $\mathrm{X}$-axis $(\mathrm{X})$. Linear relationship of gambogic acid was fine at $5-1000 \mathrm{ng} / \mathrm{mL}$. The regression equation was $Y=0.00352 X+0.02, R^{2}=$ 0.9970 . Pharmacokinetic parameters were calculated by Winnonlin 5.2 pharmacokinetic analysis software.

Data was entered and analysed by using SPSS 20.0. Measurement data were given as mean \pm standard deviation (SD). Independent samples t-test was used to review relationship of the plasma concentration and main pharmacokinetic parameters. The $p$-value of less than 0.05 was considered significant.

\section{RESULTS}

The results showed that the three batches of entrapment efficiencies were $84.12 \%, 84.46 \%$ and $85.31 \%$, respectively; the average entrapment efficiency was $84.63 \%$ for three batches. The drug loading capacity of three batches was $4.19 \%, 4.23 \%$ and $4.28 \%$, respectively; the average drug loading capacity was $4.23 \%$, showing that the process repeatability is fine.

The particle size of GNA-NLC obtained in this experiment was $146.35 \pm 1.72 \mathrm{~nm}$ for three batches and the polydispersity coefficient $(\mathrm{PI})$ was $0.26 \pm 0.02$ for three 
batches, indicating small particle size and even distribution of GNA-NLC obtained in this experiment.

The zeta potential of GNA-NLC measured by Zetasizer was $-28.24 \pm 0.13 \mathrm{mV}$ for three batches. Analysing from electrostatic repulsion, the resulting nanodispersion system has good physical stability.

The melting point of the GNA raw material was $108.1^{\circ} \mathrm{C}$, the melting peaks of GMS and SA were 61.4 and $57.3^{\circ} \mathrm{C}$, respectively; in the GNA-NLC curve, GNA melting peaks disappeared, and GMS and SA melting peaks were not seen, but a new melting peak was shown at $42.9^{\circ} \mathrm{C}$. Thus, GNA-NLC constituted a new phase, GNA no longer existed in a crystalline state, possibly distributed in the matrix in amorphous state. At the same time, the crystal lattice orderness of GMS and SA lipids decreased.

The drug-time curve of gambogic acid solution and GNA-NLC treated by tail vein injection is shown in Figure 1. The main pharmacokinetic parameters were calculated by non-compartmental model, with results shown in Table I. The results showed that the plasma concentration of GNA-NLC was significantly higher than that of the common preparation of gambogic acid $(p<0.001)$, with a half-life period of $10.14 \pm 0.03$ hours, which was 4.57 times that of gambogic acid. $\mathrm{AUC}_{0-24 \mathrm{~h}}$ of gambogic acid in GNA-NLC liposomes was 58.36 $\pm 0.23 \mu \mathrm{g} / \mathrm{h} / \mathrm{mL}$, which was 4.83 times that of gambogic acid.

Table I: Main pharmacokinetic parameters of gambogic acid and GNA-NLC in rats $(n=6$ mice).

\begin{tabular}{lcccc}
\hline Parameters & Gambogic acid & GNA-NLC & t-value & p-value \\
\hline Half-life period $(\mathrm{h})$ & $2.22 \pm 0.06$ & $10.14 \pm 0.03$ & -265.98 & $<0.001$ \\
$\mathrm{~V}(\mathrm{~mL})$ & $67.53 \pm 0.40$ & $40.31 \pm 0.23$ & 143.92 & $<0.001$ \\
$\mathrm{CL}\left(\mathrm{mL} \cdot \mathrm{h}^{1}\right)$ & $20.43 \pm 0.28$ & $3.75 \pm 0.25$ & 108.81 & $<0.001$ \\
$\mathrm{AUC}_{0-24 \mathrm{~h}}(\mu \mathrm{g} \cdot \mathrm{h} / \mathrm{mL})$ & $12.06 \pm 0.34$ & $58.36 \pm 0.23$ & -276.70 & $<0.001$ \\
\hline
\end{tabular}

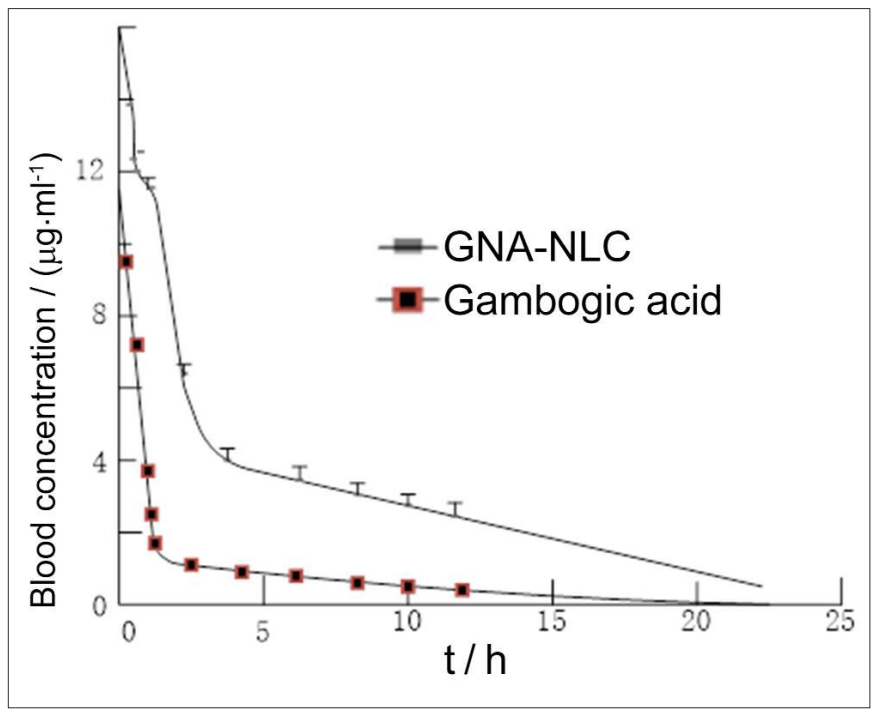

Figure 1: Drug-time curve of gambogic acid solution and GNA-NLC treated by tail vein injection.

\section{DISCUSSION}

NLC is the second generation of lipid nano-drug loading system. 9 Due to the liquid lipid loading, its entrapment efficiency and drug loading capacity are greatly improved compared with solid lipid nanoparticles. 10 GNA-NLC was successfully prepared in this experiment, with entrapment efficiency significantly improved compared to neogambogic acid solid lipid nanoparticles.

Lecithin mainly aids emulsification in the prescription. The experiment found that, entrapment efficiency and drug loading capacity increased with the increase of lecithin mass; at the same time, the particle size of the prepared nanoparticles also increased significantly. The main reason may be that lecithin as a co-emulsifier also plays the role of matrix.11 With increase of lecithin content, the matrix increased as the loaded drugs increased, so the entrapment efficiency and drug loading capacity also increased.12 On the other hand, when the matrix concentration was increased, the viscosity of the dispersed system increased and the surface tension increased correspondingly, so the diameter of the nanoparticles formed under the same emulsification conditions was large. 13

NLC belongs to the thermodynamically unstable homogeneous dispersed system, which is prone to particle size increases, flocculation, precipitation, delamination and other instabilities because of collision of molecules with each other. ${ }^{14}$ Zeta potential is one of NLC property characterisations, also an indicator of long-term stability. ${ }^{15}$ Analysing from electrostatic repulsion theory, the colloidal system is in a stable state when the absolute value of zeta potential is greater than the critical value 30.16 However, many hydrophilic emulsifiers in NLC exert a steric stabilising effect due to their special structure, which can stabilize the colloidal system with lower potential. ${ }^{17}$

Micro-column centrifugation is mainly used for the separation and purification of biological macromolecules. ${ }^{18}$ This micro-column centrifugation is mainly used for the separation and purification of biological macromolecules. Micro-column centrifugation is mainly used for the separation and purification of biological macromolecules. Method utilises the dual action of gel molecular sieve and centrifugal force to achieve rapid separation, which is currently mainly used for the purification of liposomes.19,20 The experimental results show that this method is simple, fast, reproducible, and can be used for the purification of NLC nanoparticles. During the experiment, it was found that more foam was produced at a stirring speed of $1200 \mathrm{r} /$ minute, which may affect the full contact of surfactant and oil phase, thus affecting the emulsification effect. Hence, $1000 \mathrm{r} /$ minute stirring speed was adopted in this experiment.

In this experiment using F68, polysorbate-80 and lecithin composite emulsifier, it was found that NLC prepared 
using composite emulsifier had better stability and entrapment efficiency, which was consistent with the literature reports. 21

The pharmacokinetics results in rats showed that the GNA-NLC plasma concentration was significantly higher than that of the common preparation of gambogic acid, with a half-life period of $10.14 \pm 0.03$ hours, which was 4.57 times that of gambogic acid. $\mathrm{AUC}_{0 \sim 24 \mathrm{~h}}$ of gambogic acid in GNA-NLC liposomes was $58.36 \pm 0.23 \mu \mathrm{g} / \mathrm{h} / \mathrm{mL}$, 4.83 times that of gambogic acid. It indicated that GNANLC can effectively prolong the drug's retention time in blood and maintain a high plasma concentration, suggesting that GNA-NLC can increase the anti-tumor activity of gambogic acid.

\section{CONCLUSION}

GNA-NLC can be prepared successfully by emulsification evaporation-low temperature solidification. The process is simple and easy to control. The GNA-NLC has a long cycle, and high blood concentration, sustained release compared with the raw material gambogic acid, with a good clinical application prospect.

Acknowledgements: This study was supported by the Scientific Research Project of Heilongjiang Health Planning Committee (No.2016-370); Scientific Research Projects for Basic Scientific Research in Heilongjiang Provincial Universities (No.zs201517); Natural Science Foundation of Heilongjiang Province (No.H2016090); University Nursing Program for Young Scholars with Creative Talents in Heilongjiang Province (No.UNPYSCT2017172).

\section{REFERENCES}

1. Song JZ, Yip YK, Han QB, Qiao CF, Xu HX. Rapid determination of polyprenylated xanthones in gamboge resin of Garcinia hanburyi by HPLC. J Sep Sci 2007; 30:304-9.

2. Chen F, Zhang XH, Hu XD, Zhang W, Lou ZC, Xie LH, Liu PD, Zhang $\mathrm{HQ}$. Enhancement of radiotherapy by ceria nanoparticles modified with neogambogic acid in breast cancer cells. Int J Nanomedicine 2015; 10:4957-69.

3. Zhao L, Guo QL, You QD, Wu ZQ, Gu HY. Gambogic acid induces apoptosis and regulates expressions of Bax and Bcl-2 protein in human gastric carcinoma MGC-803 cells. Biol Pharm Bull 2004; 27:998-1003.

4. Gu Q, Chen W, Duan F, Ju R. Fabrication of a nano-drug delivery system based on layered rare-earth hydroxides integrating drug-loading and fluorescence properties. Dalton Trans 2016; 45:12137-43.

5. Phatak AA, Chaudhari PD. Development and evaluation of nanostructured lipid carrier (NLC) based topical delivery of an anti-inflammatory drug. J Pharm Res 2013; 7:677-85.

6. Thomas N, Müllertz A, Graf A, Rades T. Influence of lipid composition and drug load on the in vitro performance of selfnanoemulsifying drug delivery systems. J Pharm Sci 2012; 101:1721-31.
7. Ainai A, Gupta PK. Effect of arginine hydrochloride and hydroxypropyl cellulose as stabilizers on the physical stability of high drug loading nanosuspensions of a poorly soluble compound. Int J Pharm 2008; 351:282-8.

8. Di VG, Hermida L, Batlle A, Fukuda H, Defain MV, Mamone L, et al. Characterisation of liposomes containing aminolevulinic acid and derived esters. J Photochem Photobiol B 2008; 92:1-9.

9. Yang C, Lv J, Lv T, Pan Y, Han Y, Zhao S, et al. Metal ionassisted drug-loading model for novel delivery system of cisplatin solid lipid nanoparticles with improving loading efficiency and sustained release. J Microencapsul 2016; 33: 292-8.

10. Rabanel JM, Faivre J, Paka GD, Ramassamy C, Hildgen P, Banquy $X$. Effect of polymer architecture on curcumin encapsulation and release from PEGylated polymer nanoparticles: toward a drug delivery nano-platform to the CNS. Eur J Pharm Biopharm 2015; 96:409-20.

11. Shi Q, Fan Q, Ye W, Hou J, Wong SC, Xu X, et al. Binary release of ascorbic acid and lecithin from core-shell nanofibers on blood-contacting surface for reducing long-term hemolysis of erythrocyte. Colloids Surf B Biointerfaces 2015; 125:28-33.

12. Rigolle A, Gheysen L, Depypere F, Landuyt A, Koen VDA, Foubert I. Lecithin influences cocoa butter crystallization depending on concentration and matrix. Eur J Lipid Sci Tech 2015; 117:1722-32.

13. Walesa R, Man D, Engel G, Siodlak D, Kupka T, Ptak T, et al. The impact of model peptides on structural and dynamic properties of egg yolk lecithin liposomes - experimental and DFT studies. Chem Biodivers 2015; 12:1007-24.

14. Albekery MA, Alharbi KT, Alarifi S, Ahmad D, Omer ME, Massadeh $\mathrm{S}$, et al. Optimization of a nanostructured lipid carriers system for enhancing the biopharmaceutical properties of valsartan. Dig J Nanomater Bios 2017; 12:381-9.

15. Nagaich U, Gulati N. Nanostructured lipid carriers (NLC) based controlled release topical gel of clobetasol propionate: design and in vivo characterization. Drug Deliv Transl Res 2016; 6: 289-98.

16. Khademi M, Wang WM, Reitinger W, Barz DP. The zeta potential of pmma in contact with aqueous electrolytesurfactant solutions. Langmuir 2017; 33:10473-82.

17. Joshi M, Patravale V. Nanostructured lipid carrier (NLC) based gel of celecoxib. Int J Pharm 2008; 346:124-32.

18. Ang KM, Yeo LY, Hung YM, Tan MK. Amplitude modulation schemes for enhancing acoustically-driven microcentrifugation and micromixing. Biomicrofluidics 2016; 10:261-40.

19. Yu X, Xu H, Hu M, Luan X, Wang K, Fu Y, et al. Ginsenoside Rg3 bile salt-phosphatidylcholine-based mixed micelles: design, characterization, and evaluation. Chem Pharm Bull (Tokyo) 2015; 63:361-8.

20. Cui T, Guo W. Determination of entrapment efficiency of capecitabine liposomes by microcolumn centrifugation-HPLC. Chin J Pharmaceuticals 2012;43:682-4.

21. Rizwanullah M, Ahmad J, Amin S. Nanostructured lipid carriers: a novel platform for chemotherapeutics. Curr Drug Deliv 2016; 13:4-26. 